\title{
Application of a novel cell-permeable peptide-driven protein delivery in mouse blastocysts
}

\author{
Sojung Kwon ${ }^{\dagger}$, Areum Kwak, Hyejin Shin, Soyoung Choi, Soohyun Kim and Hyunjung Jade Lim \\ Department of Biomedical Science and Technology, Institute of Biomedical Science \& Technology, \\ Konkuk University, 120 Neungdong-ro, Gwangjin-gu, Seoul 143-701, Republic of Korea
}

Correspondence should be addressed to H J Lim; Email: hlim@konkuk.ac.kr

${ }^{\dagger}$ (S Kwon is now at Priority Center, Basic Research Center, CHA University, Seoul, Republic of Korea)

\begin{abstract}
Cell-permeable peptides (CPPs) mediate the delivery of macromolecules into cells. However, whether CPPs are usable in mammalian oocytes and embryos for the modulation of protein expression has not been widely investigated. We have previously designed a novel 12-mer CPP from the conserved region of the human papillomavirus L1 capsid protein. In this study, we tested whether this peptide, LDP12, effectively delivers a protein cargo to mouse oocytes and preimplantation embryos. We prepared a LDP12-EGFP fusion protein having LDP12 as an N-terminal tag. This fusion protein readily enters HeLa cells, a cervical cancer cell line. The entry of LDP12-EGFP was partially blocked by amiloride, while cytochalasin D or methyl- $\beta$-cyclodextrin slightly increased the uptake. LDP12-EGFP shows efficient transduction in mouse blastocysts, but not in oocytes, two-cell-stage, or morula-stage-preimplantation embryos. LDP12mediated delivery of EGFP-LC3, a widely used marker of autophagic activation, is successful in HeLa cells and mouse blastocysts, as it enters cells and exhibits a signature punctate pattern. The lipidation of EGFP-LC3 also normally occurs after transduction, suggesting that the transduced protein retains the functional characteristics. Collectively, we show that LDP12-driven protein delivery is a fast and convenient method applicable to mouse blastocysts and reproductive cancer cells.
\end{abstract}

Reproduction (2013) $\mathbf{1 4 6} 145-153$

\section{Introduction}

Cell-permeable peptides (CPPs) are widely used to mediate the delivery of macromolecules into cells. CPPs generally contain several basic amino acids, and this characteristic is considered to aid in the penetration of the lipid bilayer of cells (van den Berg \& Dowdy 2011). The protein transduction domain (PTD) is the first reported CPP, which is a short peptide derived from the TAT protein of the human immunodeficiency virus (HIV). PTD-tagged fusion proteins readily enter cells in vitro and in vivo (Schwarze et al. 1999). The entry of CPPs into cells depends on various endocytic pathways (Fittipaldi et al. 2003, Nakase et al. 2004, Wadia et al. 2004, Kwon et al. 2009). Endocytosis inhibitors, such as amiloride, methyl- $\beta$-cyclodextrin $(M \beta C D)$, cytochalasin $D$, and mutant forms of the key molecules of endocytic pathways, are generally used to delineate which endocytic pathway is used by a specific CPP (Kwon et al. 2009).

CPP-mediated protein delivery has been applied to various cell systems including embryonic stem cells and induced pluripotent stem cells (Peitz et al. 2007,
Tang et al. 2011, Jo et al. 2012, Zhang et al. 2012). The key advantage of modulating protein expression using CPPs is that the genetic profiles of the target cells are not altered. This is crucial especially for germ cells or embryos, because the altered genetic makeup caused by viral vectors may cause unintended developmental defects. The above-mentioned PTD system has been successfully used in one-cell- or two-cell-stage preimplantation mouse embryos, in which the PTD-CRE recombinant protein enters blastomeres and results in CRE-mediated excision (Kim et al. 2009). Regarding mammalian oocytes, the usage of CPPs has not been investigated much. We previously reported that MPG, a designed CPP (Morris et al. 1997), enters mouse oocytes and stays in the periphery when used as an $\mathrm{N}$-terminal tag of EGFP (Kwon et al. 2009). However, the efficiency of CPP-mediated delivery in oocytes seems to be limited, and this may be partly due to the presence of numerous cortical granules at the periphery of oocytes. These granules are densely localized at the periphery of oocytes and are known to exocytose at the time of fertilization (Ducibella 1996). Thus, the presence of these granules may be associated with the limited 
transduction efficiency of CPP-mediated cargo delivery in oocytes.

Human papillomaviruses (HPVs) are non-enveloped DNA viruses that target stratified epithelial tissues in skin and the female reproductive tract (Sasagawa et al. 1995). L1 protein is the major capsid protein (Joyce et al. 1999). We have recently reported a novel CPP designed from the conserved carboxy-terminal region of the HPV type 11 L1 protein (Lee \& Lim 2012). This 12-mer peptide, LDP12, itself possesses the ability to enter various cells within several minutes in vitro (Lee \& Lim 2012). Intraluminal delivery of a FITC-conjugated LDP12 peptide into mouse uterus also leads to uptake by uterine cells (Lee \& Lim 2012). In the present study, we sought to investigate whether LDP12 can be used as a delivery tag for protein cargo and whether this system is applicable to preimplantation mouse embryos and oocytes. We also performed a functional application test using LDP12-EGFP-LC3, a widely used marker of autophagic activation (Kabeya et al. 2000). Herein, we report that the LDP12-tagged fusion protein is an effective and easy option for the delivery of proteins into reproductive cancer cells and mouse blastocysts.

\section{Materials and methods Cell culture}

HeLa human cervical cancer cells were purchased from the American Type Culture Collection (Rockville, MD, USA) and were cultured in DMEM or RPMI supplemented with $10 \%$ FBS (Invitrogen).

\section{Materials \\ Cytochalasin D, amiloride, and M $\beta C D$ were purchased from Sigma-Aldrich and were used at the indicated concentrations. FM4-64, a live dye for endosomes, was purchased from Invitrogen.}

\section{Mouse and oocyte/embryo handling}

Mice were maintained in accordance with the policies of the Konkuk University Institutional Animal Care and Use Committee. The mice were housed in a controlled barrier facility in Konkuk University. Cumulus-oocyte complexes (COCs) were isolated from 4-week-old ICR mice (Orient-Bio, Kyunggi-do, Korea). Cumulus cells were removed by gentle pipetting, and zona pellucidae were digested with acidic Tyrode solution (Kwon et al. 2009). Metaphase I- or metaphase II-stage oocytes were used in the experiments. Two-cell-stage embryos were obtained by oviduct flushing on day 2 of pregnancy (vaginal plug, day 1 of pregnancy) and were cultured in KSOM-AA media (Millipore, Billerica, MA, USA).

\section{Expression and purification of LDP12 fusion proteins}

The nucleotide sequence of LDP12 (TAPKRKRTKTKK, derived from the carboxy terminus of the HPV type 11 L1) (Lee \& Lim 2012) was included in the $5^{\prime}$ primer and was used to directly amplify the EGFP coding sequence using pET15b-EGFP (Novagen, Madison, WI, USA) as a template. The PCR product was cloned into the $\mathrm{Ndel}$ and BamHI sites of the pET15b vector. Primers used were $C$ AGC CAT ATG ACT GCC CCT AAA CGT AAG CGC ACC AAA ACT AAA AAG GTG AGC AAG GGC GAG GAG CTG TTC ACC GGG GTG GTG CCC (up, LDP12 sequence is underlined) and AGCC GG ATC C TTA CTT GTA CAG CTC GTC CAT (down). The LDP12-EGFP-LC3 construct was cloned into the pProEx/HTa vector (Invitrogen), and the mRFP-EGFP-LC3 plasmid (Kimura et al. 2007) was used as a template for PCR. Purification of CPP-fused recombinant proteins was performed as described previously (Kwon et al. 2009). Ni-NTA agarose resin ( $1 \mathrm{ml}, 50 \% \mathrm{w} / \mathrm{v}$ slurry, Qiagen) was used to purify proteins from the lysate following the manufacturer's protocol.

\section{Flow cytometric analysis}

HeLa cells treated with proteins were washed with PBS (Invitrogen) and the acid wash buffer $(0.2 \mathrm{M}$ glycine and $0.15 \mathrm{M} \mathrm{NaCl}, \mathrm{pH} 3.0$ ) for $30 \mathrm{~s}$. The acid wash eliminates nonspecific binding to the cell surface (Kameyama et al. 2007). The cells were then trypsinized with a $0.25 \%$ trypsin-EDTA solution for $10 \mathrm{~min}$. The cells were resuspended in DMEM supplemented with $10 \%$ FBS and were washed with PBS containing 3\% FBS. After washing, the cells were analyzed using a FACSCalibur flow cytometer (BD Biosciences, Franklin Lakes, NJ, USA) equipped with the Cell Quest Pro software (BD Biosciences).

\section{Confocal microscopy}

The cells were seeded into a glass-bottomed confocal dish (SPL Lifesciences, Pocheon, Korea) at a density of $1 \times 10^{5}$ one day before the experiments. Protein-treated cells were washed with HBSS, acid wash buffer, and then again with HBSS. The cellular uptake of the EGFP fusion proteins was visualized in HBSS using an Olympus FV1000 spectral confocal microscope (Olympus, Tokyo, Japan) equipped with a warm plate. In some experi-

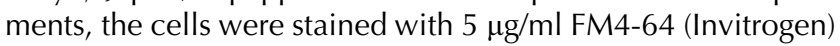
for $1 \mathrm{~min}$. Live images were captured by confocal microscopy.

\section{Inhibition of endocytosis}

Cytochalasin D, amiloride, or M $B C D$ was used at the indicated concentrations to block a specific endocytic pathway (Kwon et al. 2009). The cells were treated with each inhibitor $30 \mathrm{~min}$ prior to the addition of the LDP12-EGFP fusion protein. After $1 \mathrm{~h}$, the cells were acid washed and subjected to flow cytometric analyses.

\section{Protein preparation and western blotting}

HeLa cells $\left(2 \times 10^{5}\right)$ were plated onto a six-well plate and were treated with the LDP12-EGFP-LC3 protein at $100 \mu \mathrm{g} / \mathrm{ml}$. 
After acid washing, the cells were directly lysed in SDS sample buffer $(150 \mu \mathrm{l})$. The lysates were then centrifuged at $12600 \mathrm{~g}$ for $3 \mathrm{~min}$. After boiling, the supernatant $(30 \mu \mathrm{l})$ was loaded onto a $12 \%$ SDS-polyacrylamide gel and blotted onto PVDF membranes (Millipore). The membranes were blocked with $5 \%$ skim milk in TBS for $1 \mathrm{~h}$ and incubated with anti-GFP (1:2000, Abfrontier, Seoul, Korea) or anti-LC3 (1:2000, Abcam, Cambridge, UK) antibody overnight at $4{ }^{\circ} \mathrm{C}$. HRP-conjugated goat anti-rabbit antibody (GeneDEPOT, Barker, TX, USA) was used at a 1:10 000 dilution. Super Signal West Femto ECL reagent (Thermo Scientific, Wilmington, DE, USA) was used. The chemiluminescence signal was detected using a LAS3000 system (FUJIFILM, Tokyo, Japan).

\section{Results}

\section{Efficient transduction of the LDP12-EGFP fusion protein into HeLa cells}

We prepared a fusion protein construct as shown in Fig. 1A. The LDP12 sequence was cloned in frame at the $\mathrm{N}$-terminus of the EGFP coding sequence. MPG is a designed CPP (Morris et al. 1997), and MPG-EGFP is a cell-permeable fusion protein with proven efficiency (Kwon et al. 2009). We used MPG-EGFP fusion protein as a positive control in some experiments. EGFP without a CPP tag was used as a negative control. HeLa cells were treated with purified proteins for $1 \mathrm{~h}$
A

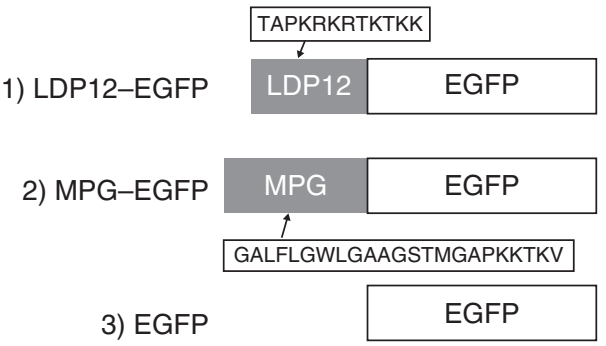

B

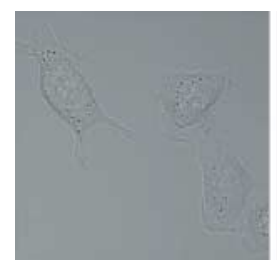

C
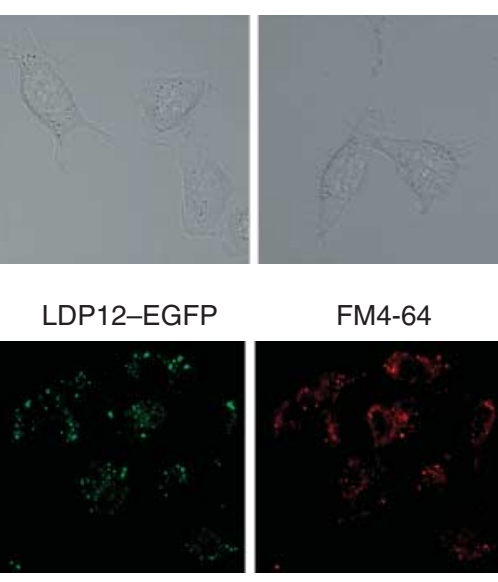

FM4-64
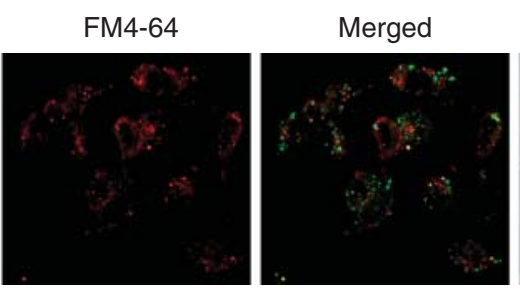

D
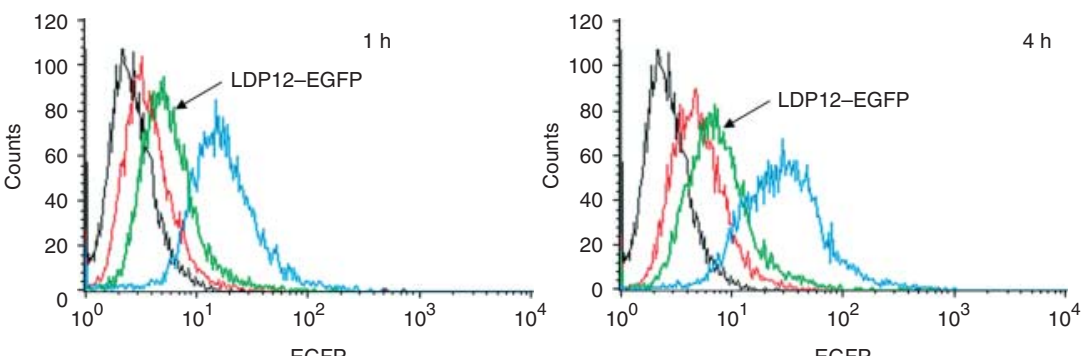

\begin{tabular}{|r|c|c|}
\hline \multicolumn{2}{|c|}{ EGFP } \\
\hline Color - group & $1 \mathrm{~h}(\%)$ & $4 \mathrm{~h}(\%)$ \\
\hline Black: control & 0.1 & 0.1 \\
\hline Red: EGFP & 4.7 & 16.7 \\
\hline Green: LDP12-EGFP & 24.6 & 53.4 \\
\hline Blue: MPG-EGFP & 96.9 & 98.2 \\
\hline
\end{tabular}

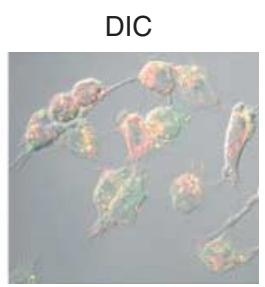

MPG-EGFP

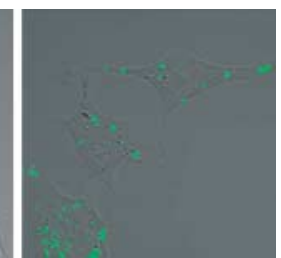

Figure 1 Efficient transduction of the LDP12-EGFP fusion protein into HeLa cells. (A) Fusion protein constructs used in this study. LDP12 (12 mer) or MPG (24 mer) sequence was added to the EGFP coding sequence as an $\mathrm{N}$-terminal tag. The MPG-EGFP fusion protein was used as a positive control in some experiments (Kwon et al. 2009). (B) Confocal live imaging of HeLa cells treated with EGFP, LDP12-EGFP, or MPG-EGFP. The cells were treated with $40 \mu \mathrm{g} / \mathrm{ml}$ MPG-EGFP, $100 \mu \mathrm{g} / \mathrm{ml}$ EGFP, or LDP12-EGFP for $1 \mathrm{~h}$. The cells were acid washed. Photomicrographs were taken at $40 \times$ magnification and were zoomed in $3 \times$. (C) Fluorescence imaging of $4 \%$ paraformaldehyde (PFA)-fixed HeLa cells. The cells were co-stained with FM4-64, a general endosomal marker. LDP12-EGFP was added at $100 \mu \mathrm{g} / \mathrm{ml}$. EGFP signal is shown in green and FM4-64 in red. Overlapping signals generated yellow fluorescence. FM4-64 was used at $5 \mu \mathrm{g} / \mathrm{ml}$ for $3 \mathrm{~min}$. Photomicrographs were taken at $60 \times$ magnification and were zoomed in $2 \times$. (D) Time-dependent increases of the cellular uptake rate of LDP12-EGFP. EGFP $(100 \mu \mathrm{g} / \mathrm{ml})$, MPG-EGFP $(40 \mu \mathrm{g} / \mathrm{ml})$, or LDP12-EGFP $(100 \mu \mathrm{g} / \mathrm{ml})$ was added to HeLa cells, and the rate of cellular uptake was assessed by flow cytometric analyses at 1, 2, or $4 \mathrm{~h}$. The cells were acid washed and trypsinized for FACS analysis. At 2-h incubation, $45.5 \%$ of the cells were LDP12-EGFP positive (data not shown). These experiments were repeated at least three times with similar results. 
and were washed with the acid buffer to remove molecules attached by non-specific cell surface binding (Kameyama et al. 2007). By confocal live imaging, both LDP12-EGFP and MPG-EGFP were shown to be internalized within $1 \mathrm{~h}$ and to exhibit small dot patterns in the cytoplasm (Fig. 1B). FM4-64 is a live cell dye that stains endosomal vesicles in red (Kwon et al. 2009). When LDP12-EGFP (green) and FM4-64 (red) were added together to HeLa cells, a subset of vesicles exhibited an overlapping signal (yellow). Thus, the internalized LDP12-EGFP fusion proteins seem to be partly located in the FM4-64-positive endosomal vesicular structures. Signals of some vesicles did not overlap, suggesting that the LDP12-EGFP proteins were also present in the non-endosomal vesicular structures. We quantified the cellular uptake rate of LDP12-EGFP by flow cytometry. Within $1 \mathrm{~h}$ of treatment, about $25 \%$ of the HeLa cells were determined to be EGFP positive. This rate increased up to $53 \%$ by $4 \mathrm{~h}$, suggesting a gradual increase in the cellular uptake of LDP12-EGFP.
This was in contrast to MPG-EGFP, which exhibited $97 \%$ cellular uptake by $1 \mathrm{~h}$ (Fig. 1D). Distinct temporal kinetics of transduction suggests a different mechanism of entry used by these CPP-tagged proteins.

\section{Effects of endocytosis inhibitors on the transduction of LDP12-EGFP}

Multiple mechanisms are used during endocytosis, such as clathrin-mediated, caveolae-mediated, and lipid raftmediated endocytosis and macropinocytosis (Conner \& Schmid 2003). Lipid raft-mediated macropinocytosis, the cell-drinking process, is used by several known CPPs, such as HIV PTD and polyarginine (Fittipaldi et al. 2003, Nakase et al. 2004, Wadia et al. 2004). Arrays of inhibitors can be used to delineate which pathway is operative during cellular uptake by a CPP (Kwon et al. 2009). Amiloride, a sodium channel blocker, inhibits macropinocytosis. M $\beta C D$ depletes cholesterol from the plasma membrane and is used as an inhibitor of
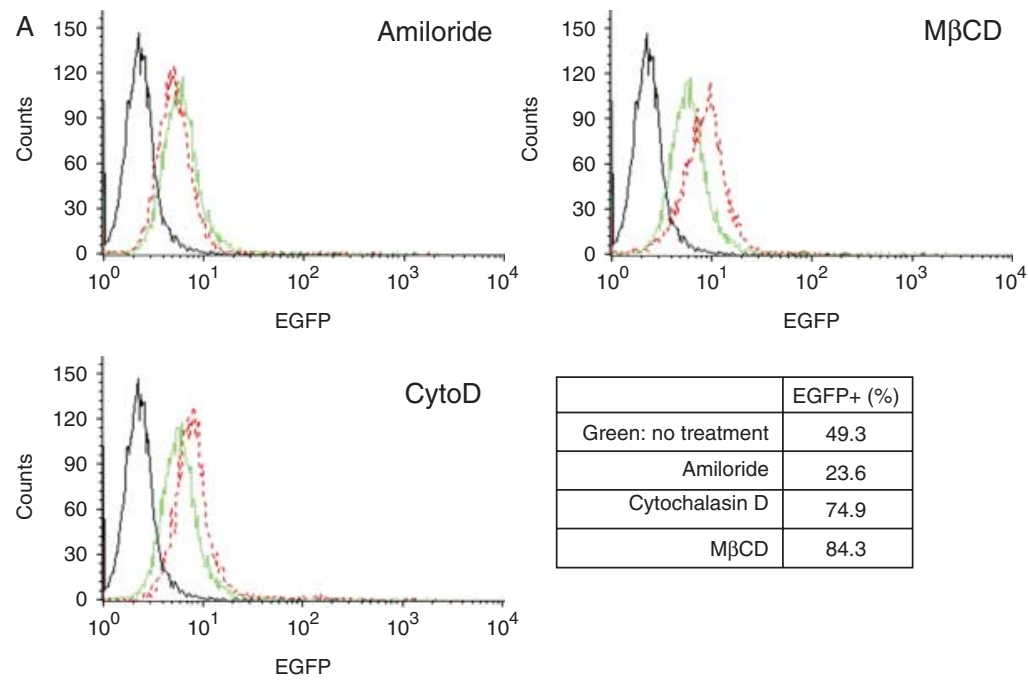

Fixed HeLa cells

B
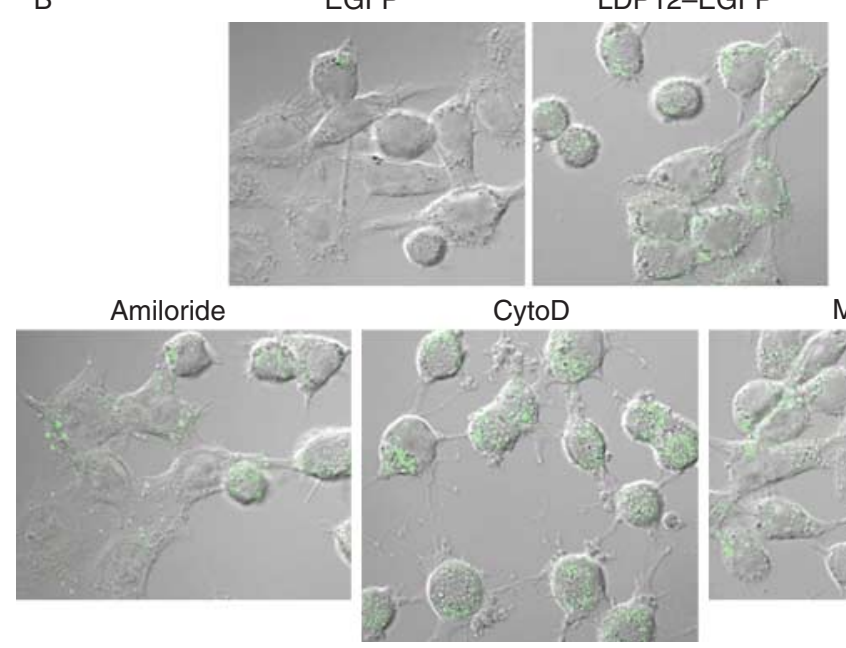

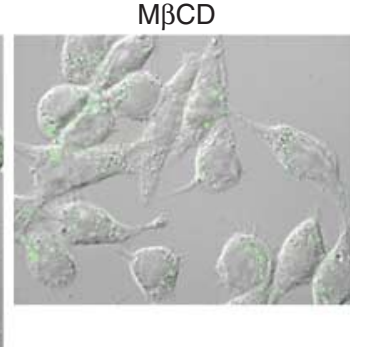

Figure 2 Transduction of LDP12-EGFP is influenced by endocytic inhibitors. (A) Flow cytometric analysis of LDP12-EGFP uptake in the presence of various endocytosis inhibitors. An inhibitor or DMSO (vehicle, $0.2 \%$ ) was added to HeLa cells 30 min prior to the addition of EGFP or LDP12-EGFP fusion protein (1 h). All the cells were washed with the acid buffer before FACS analysis. The following concentrations were used: $10 \mu \mathrm{M}$ cytochalasin D, $4 \mathrm{mM}$ amiloride, and $5 \mathrm{mM} M \beta C D$. Green dashed line, no treatment; red dotted line, inhibitor added. These experiments were repeated at least three times with similar results, and one representative set of data is shown. (B) Confocal images of $4 \%$ PFA-fixed HeLa cells treated with an endocytosis inhibitor as indicated. Photomicrographs were taken at $40 \times$ magnification and were zoomed in $3 \times$. CytoD, cytochalasin D; $M \beta C D$, methyl- $\beta$ cyclodextrin. 
A

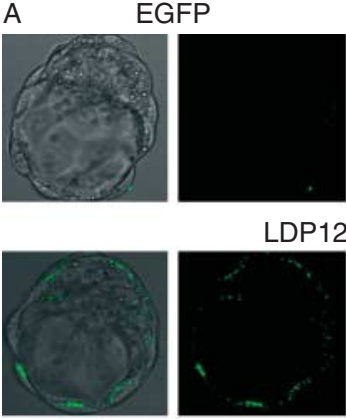

Amiloride

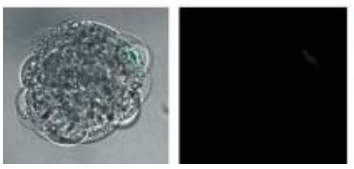

MPG-EGFP

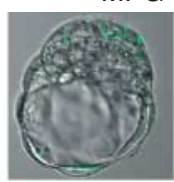

-EGFP
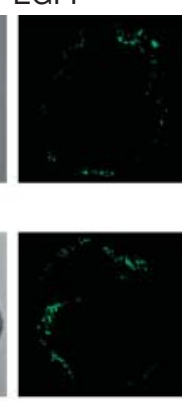

CytoD

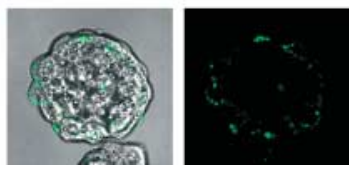

B

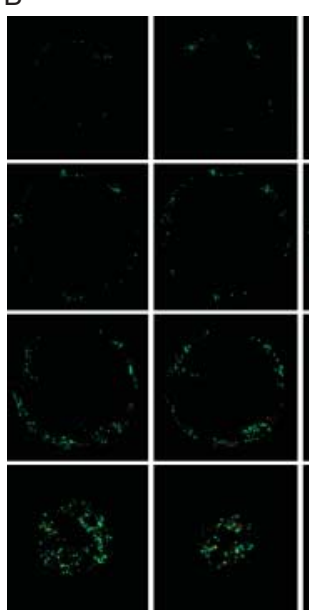

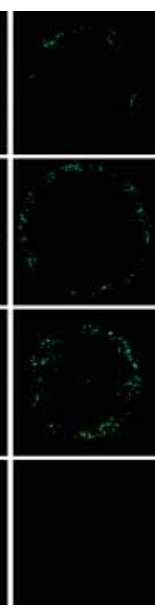

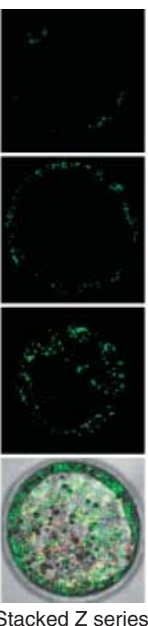

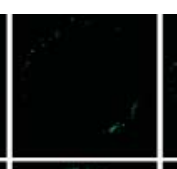
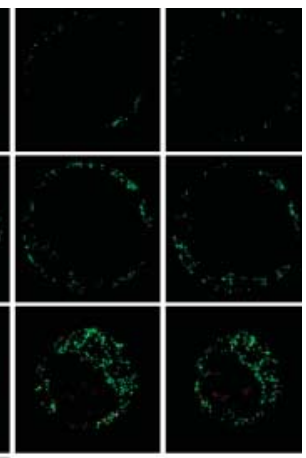

Figure 3 Efficient transduction of LDP12-EGFP into mouse blastocysts. (A) Day 4 blastocysts were obtained from day 4 pregnant mice and were cultured in KSOM-AA media in the presence of $40 \mu \mathrm{g} / \mathrm{ml}$ MPG-EGFP or $100 \mu \mathrm{g} / \mathrm{ml}$ LDP12-EGFP fusion protein for $1 \mathrm{~h}$. Live images of blastocysts were observed under a confocal microscope. Green fluorescence indicates the internalized fusion proteins. Photomicrographs were taken at $40 \times$ magnification and were zoomed in $3 \times$. CytoD, cytochalasin D. (B) Cellular distribution of internalized LDP12-EGFP in mouse blastocysts. To confirm that LDP12-EGFP uptake can be observed in both the inner cell mass and trophectodermal cells, Z series images of $3 \mu \mathrm{m}$ step size (channel 1, GFP; channel 2, Cy5.5) were obtained from a single blastocyst. Zona-encased blastocysts were treated with 100 $\mu$ g/ml EGFP or LDP12-EGFP for $1 \mathrm{~h}$. Embryos were also counter-stained with FM4-64 for 3 min. This experiment was performed using four different blastocysts, and one representative set of images is shown.

caveolae-mediated endocytosis (Hao et al. 2004). Cytochalasin D, an actin-depolymerizing drug, inhibits actin-mediated macropinocytosis (West et al. 1989). Flow cytometric analyses showed that amiloride partially inhibited LDP12-EGFP uptake, while both cytochalasin $D$ and $M \beta C D$ increased the transduction rate (Fig. 2A). The amiloride-sensitive nature of LDP12-EGFP is similar to that of MPG-EGFP, which exhibited partial amiloride sensitivity (Kwon et al. 2009). Our finding that cytochalasin D or M $B C D$ treatment significantly increases the transduction of LDP12-EGFP suggests that the blockage of certain endocytic pathways somehow increases the endocytic rate of LDP12-EGFP. HeLa cells treated with each inhibitor are shown in Fig. 2B. Our results suggest that the internalization of LDP12-EGFP may have occurred through more than one endocytic pathway.

\section{Efficient entry of LDP12-EGFP into mouse blastocysts}

We tested whether the LDP12-driven protein delivery system is applicable to preimplantation mouse embryos. Day 4 blastocysts have two different cell types: the inner cell mass contributing mostly to the embryo proper and the trophectoderm contributing to the extraembryonic cell lineages (Wang \& Dey 2006). Blastocysts were obtained from day 4 pregnant mice and were cultured for $1 \mathrm{~h}$ in KSOM-AA media with $100 \mu \mathrm{g} / \mathrm{ml}$ LDP12-EGFP. As shown in Fig. 3A, live imaging of GFP signal showed that LDP12-EGFP was internalized in both the inner cell mass and trophectodermal cells. This is clearly visible in Fig. 3B with $\mathrm{Z}$ series sectional images. Amiloride treatment for
30 min affected embryonic morphology and also hindered LDP12-EGFP uptake (Fig. 3A). Blastocysts treated with cytochalasin $\mathrm{D}$ exhibited blastocoel shrinkage, but LDP12-EGFP uptake seemed to be unaffected. M $\beta C D$ treatment resulted in detrimental effects on blastocysts as reported previously (Comiskey \& Warner 2007); thus, its effect on LDP12-EGFP uptake could not be assessed (data not shown). We also tested whether LDP12-EGFP was internalized in mouse oocytes or earlier-stage embryos. Regardless of the presence of zona pellucida, this protein was not found to be internalized in oocytes or two-cell-stage embryos (Fig. 4).

\section{Functional application of the LDP12 system: LDP12-EGFP-LC3 as a tool to monitor autophagy}

The LDP12 fusion protein works well in mouse blastocysts, and we further examined the application of the LDP12 system with a functionally testable protein cargo. LC3 (Atg8) is an ubiquitin-like autophagy protein widely used as a monitoring marker of autophagic flux (Kabeya et al. 2000). LC3 is extensively used in a GFP-LC3 fusion protein form (Mizushima et al. 2004, 2010). The LC3-I form is modified into a phosphatidylethanolamine (PE)-conjugated form, LC3-II, in cells. The LC3-II form is known to surround the growing autophagosomes within cells, and increased LC3-II levels represent autophagic activation (Klionsky et al. 2007, Yang \& Klionsky 2010). On the SDS-PAGE gel, the LC3-II form migrates slightly faster than the LC3-I form. Generally in a cell culture system, a plasmid harboring the fusion gene of GFP-LC3 is transfected to monitor 

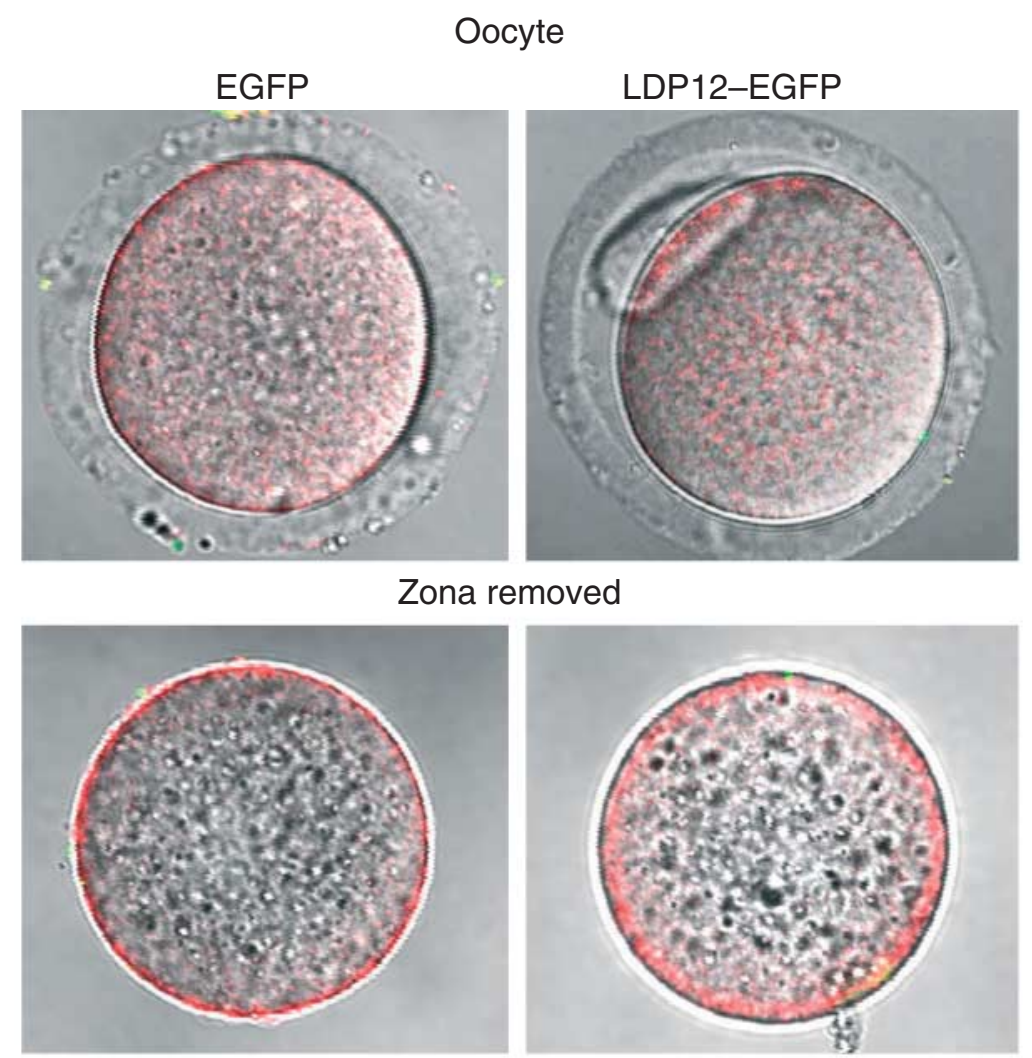

Two cell embryo
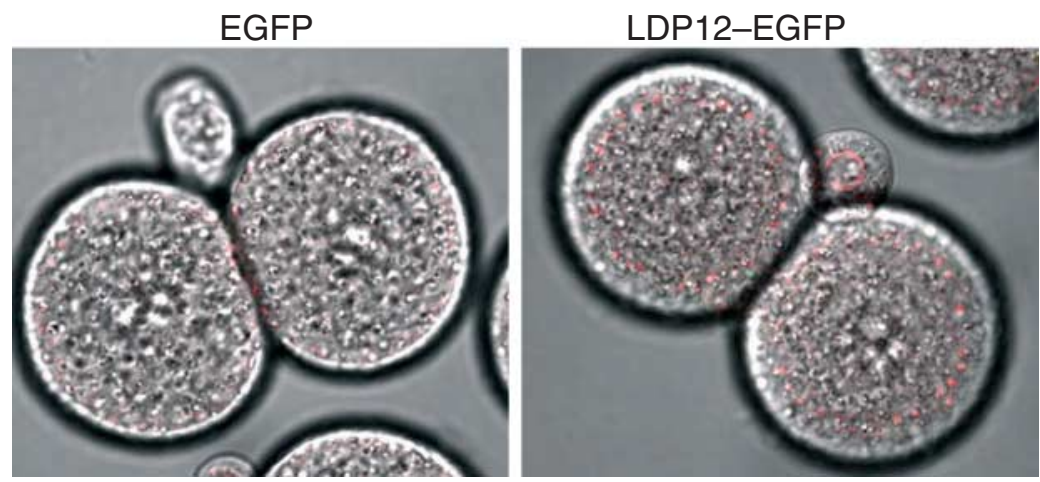

Figure 4 LDP12-EGFP is not internalized in mouse oocytes or earlier-stage preimplantation embryos. Oocytes or embryos were treated with $100 \mu \mathrm{g} / \mathrm{ml}$ EGFP or LDP12-EGFP for $1 \mathrm{~h}$. Zona pellucidae were removed using the acid Tyrode solution. Note the endosomal staining by FM4-64 (red), but no green signal can be seen in the oocytes or two-cell embryos. autophagic activation and flux (Kimura et al. 2007), but the conventional transfection method does not work well in preimplantation embryos. Thus, we designed a LDP12-EGFP-LC3 fusion protein and tested its efficacy in HeLa cells and mouse blastocysts. The fusion protein entered both HeLa cells and blastocysts within $1 \mathrm{~h}$ of incubation and exhibited a signature punctate pattern, as shown in Fig. 5A. This pattern is similar to the punctate pattern exhibited in blastocysts obtained from GFP-LC3 transgenic mice (Lee et al. 2011). We also confirmed by western blotting that the internalized LDP12-EGFP-LC3 fusion proteins undergo PE conjugation and produce LC3-II forms. Thus, two sets of doublets are shown on western blots (Fig. 5B, right panel, two brackets). The higher set is positive for both anti-GFP and anti-LC3 antibodies.
The lower set is the endogenous LC3-I (upper band) and LC3-II (lower band) in the HeLa cells. This experiment shows that not only is LDP12-EGFP-LC3 internalized, but that it is also normally processed for PE conjugation. In twocell- or morula-stage embryos, LDP12-EGFP-LC3 was not found to be internalized (Fig. 6).

\section{Discussion}

In this study, we showed that LDP12 is an efficient CPP functionally applicable to mouse blastocysts. Considering that the conventional transfection method is not efficient in blastocysts, LDP12-driven protein delivery offers a new system to manipulate protein expression in them. The internalization of LDP12-EGFP was observed 


\begin{tabular}{|c|c|c|c|}
\hline & TAPKRKRTKTKK & & \\
\hline LDP12-EGFP-LC3 & LDP12 & EGFP & LC3 \\
\hline
\end{tabular}

A
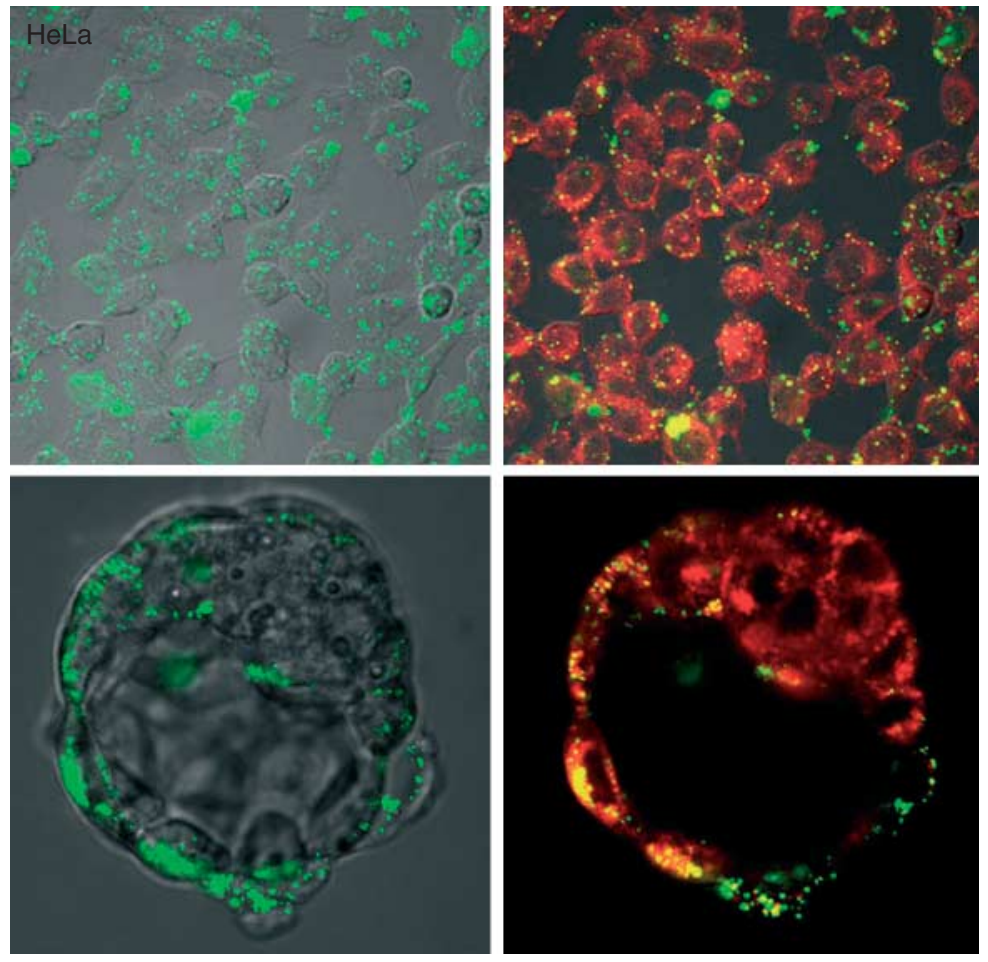

B

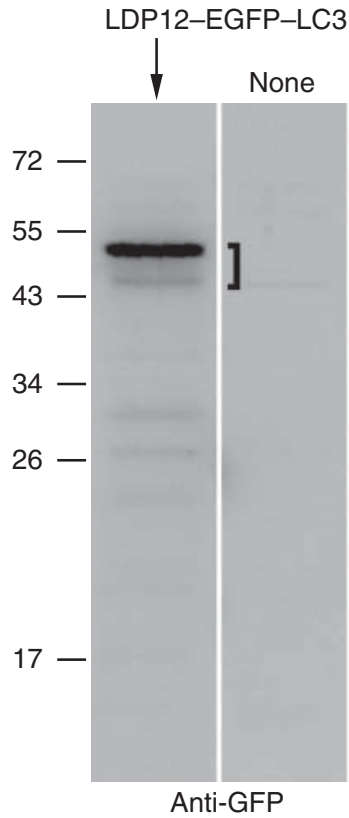

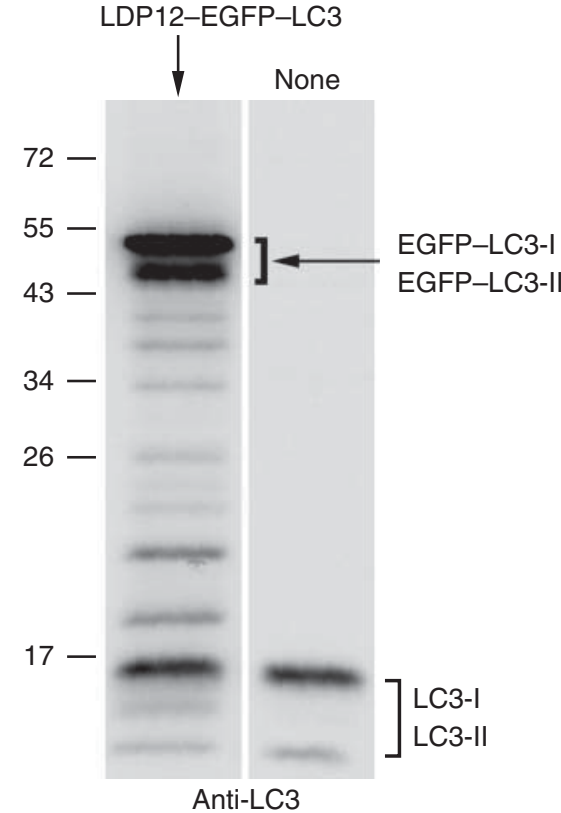

Anti-LC3
Figure 5 LDP12-driven transduction of EGFP-LC3, a marker of autophagic activation. (A) The LDP12-EGFP-LC3 fusion protein construct is shown at the top. HeLa cells or mouse blastocysts were treated with the LDP12-EGFP-LC3 protein $(100 \mu \mathrm{g} / \mathrm{ml})$. Endosomes are counter-stained with FM4-64 (red). The left panel shows the EGFP signal (green), and the right panel is an overlay of EGFP and FM4-64. (B) Western blotting showed that the internalized LDP12-EGFP-LC3 proteins undergo lipidation like the endogenous LC3 and produce the LC3-II form. HeLa cells $\left(2 \times 10^{5}\right)$ were treated with $100 \mu \mathrm{g} / \mathrm{ml}$ LDP12-EGFP-LC3 for $1 \mathrm{~h}$ and were subjected to SDS-PAGE. The cells were directly lysed in SDS sample buffer $(150 \mu \mathrm{l})$ and $30 \mu \mathrm{l}$ were loaded into each lane. Anti-GFP and anti-LC3 antibodies were used at 1:2000. None, no treatment. Upper bracket: EGFP-LC3-I and EGFP-LC3-II forms; lower bracket: endogenous LC3-I and LC3-II forms. 

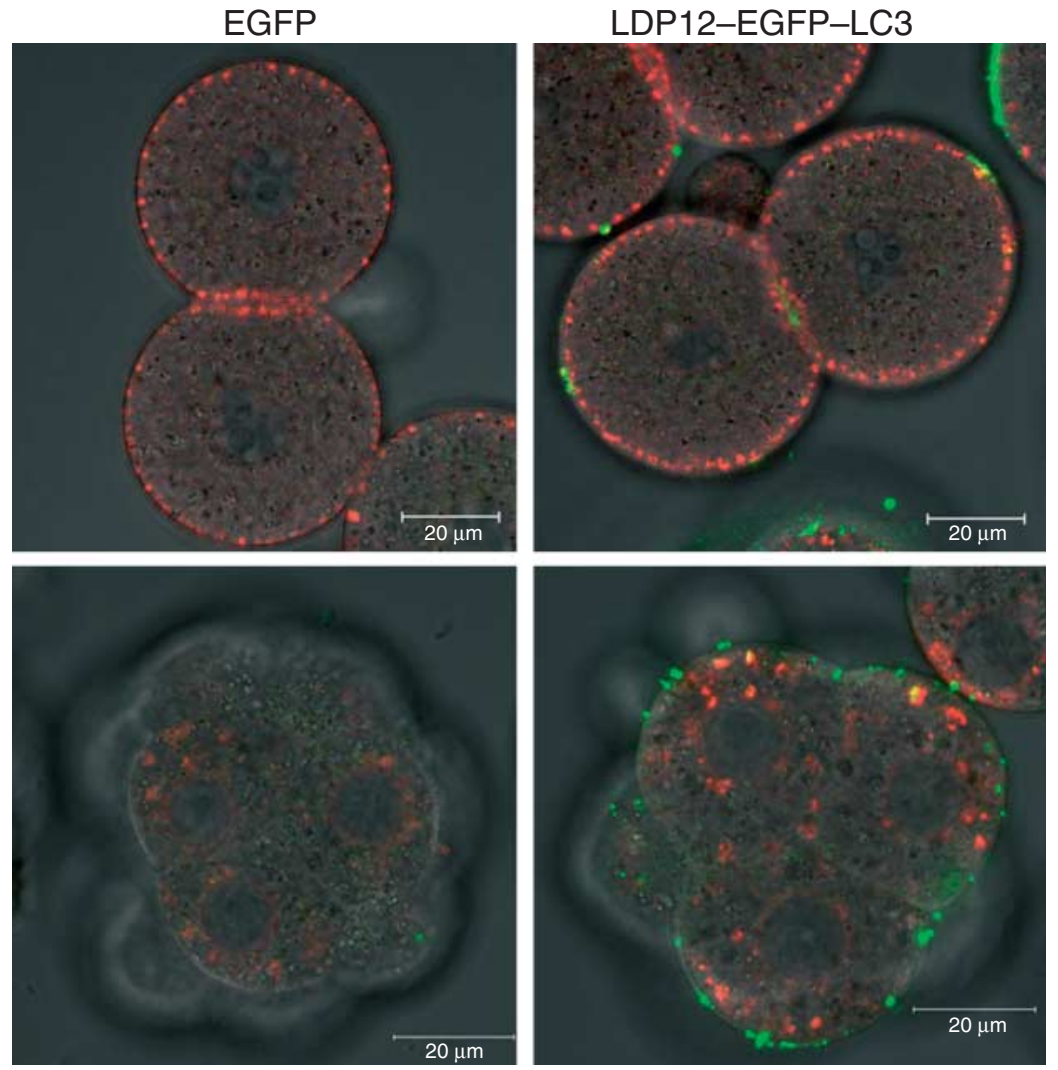

Figure 6 LDP12-EGFP-LC3 is not internalized in the earlier-stage preimplantation embryos. Embryos were treated with $100 \mu \mathrm{g} / \mathrm{ml}$ EGFP or LDP12-EGFP-LC3 for $1 \mathrm{~h}$. Zona pellucidae were removed using acidic Tyrode solution. Note the distinct endosomal structures in embryos stained by FM4-64 (red). While some green signal can be seen at the periphery of morula treated with LDP12-EGFP-LC3, it does not seem to be internalized. Scale bars, $20 \mu \mathrm{m}$. in both the inner cell mass and trophectoderm of mouse blastocysts (Fig. 3B), indicating that this system is also applicable to protein therapeutics in blastocystderived stem cells such as embryonic stem cells and trophoblast stem cells. However, the efficiency of LDP12-mediated delivery in oocytes and earlier-stage embryos seems to be limited (Fig. 4). The LDP-tagged fusion proteins that we used in this study were up to $50 \mathrm{kDa}$ in size. Whether there would be a size limitation for a protein cargo requires further investigation. It is of note that, a PTD-tagged protein cargo of about $120 \mathrm{kDa}$ has been successfully used in vivo (Schwarze et al. 1999).

The use of CPPs has not been investigated much in mouse oocytes and embryos. Considering that the manipulation of gene expression in these cells mostly involves invasive or low-efficiency techniques, such as microinjection, electroporation, and viral vector delivery, the application of CPPs will open up new ways to manipulate gene/protein expression. Only a couple of reports are available with regard to the application of CPPs in oocytes and preimplantation embryos. HIV TAT protein-derived PTD has been shown to be effective in two-cell-stage mouse embryos (Kim et al. 2009), and MPG-EGFP protein has exhibited limited distribution in mouse oocytes (Kwon et al. 2009). We repeatedly observed that LDP12-EGFP-LC3 exhibited strong surface binding on morulae, but not on the two-cell embryos (Fig. 6) or on oocytes (data not shown). This observation suggests that the changing biochemical nature of plasma membranes in early preimplantation embryos (Ferreira et al. 2012) influences the initial interaction with the LDP12 fusion protein. Presently, it is not clear what distinguishes blastocysts from earlier-stage embryos with regard to the transduction of LDP12-driven protein cargo. Further investigation of the differential preferences of preimplantation embryos for various CPPs will be useful to target embryos with selective CPPs.

The biochemical characteristics of the LDP12EGFP-LC3 protein are maintained after transduction, as its PE conjugation normally occurs after cellular entry (Fig. 5B). The presence of LDP12 tag at the $\mathrm{N}$-terminus did not seem to affect the biochemical properties of the LC3 protein. Thus, LDP12-mediated delivery of the LC3 protein is an option for monitoring autophagic activation in reproductive cancer cells and blastocysts. We have previously shown that high levels of endocannabinoids increase autophagy in mouse blastocysts (Oh et al. 2013), and immunofluorescence staining on fixed embryos has been mainly used to visualize the autophagic response. As the internalization of LDP12-EGFP-LC3 is highly successful with no lethal effects on blastocysts, it now offers a new way to visualize autophagic response without fixation. Thus, autophagy monitoring by the use of such a transient system may be useful in addressing certain stress conditions in embryos in culture, such as oxidative stress, starvation, and deprivation of specific factors and 
hormones. Whether this system would be applicable to other primary cells with low transfection efficiency warrants further investigation.

\section{Declaration of interest}

The authors declare that there is no conflict of interest that could be perceived as prejudicing the impartiality of the research reported.

\section{Funding}

This study was supported by Konkuk University in 2011.

\section{Acknowledgements}

We thank the members of the Lim laboratory for their support.

\section{References}

van den Berg A \& Dowdy SF 2011 Protein transduction domain delivery of therapeutic macromolecules. Current Opinion in Biotechnology 22 888-893. (doi:10.1016/j.copbio.2011.03.008)

Comiskey M \& Warner CM 2007 Spatio-temporal localization of membrane lipid rafts in mouse oocytes and cleaving preimplantation embryos. Developmental Biology 303 727-739. (doi:10.1016/j.ydbio.2006.12.009)

Conner SD \& Schmid SL 2003 Regulated portals of entry into the cell. Nature 422 37-44. (doi:10.1038/nature01451)

Ducibella T 1996 The cortical reaction and development of activation competence in mammalian oocytes. Human Reproduction Update $\mathbf{2}$ 29-42. (doi:10.1093/humupd/2.1.29)

Ferreira CR, Pirro V, Eberlin LS, Hallett JE \& Cooks RG 2012 Developmental phases of individual mouse preimplantation embryos characterized by lipid signatures using desorption electrospray ionization mass spectrometry. Analytical and Bioanalytical Chemistry 404 2915-2926. (doi:10.1007/s00216-012-6426-4)

Fittipaldi A, Ferrari A, Zoppe M, Arcangeli C, Pellegrini V, Beltram F \& Giacca M 2003 Cell membrane lipid rafts mediate caveolar endocytosis of HIV-1 Tat fusion proteins. Journal of Biological Chemistry 278 34141-34149. (doi:10.1074/jbc.M303045200)

Hao M, Mukherjee S, Sun Y \& Maxfield FR 2004 Effects of cholesterol depletion and increased lipid unsaturation on the properties of endocytic membranes. Journal of Biological Chemistry 279 14171-14178. (doi:10.1074/jbc.M309793200)

Jo J, Song H, Park SG, Lee SH, Ko J, Park JH, Jeong J, Cheon YP \& Lee DR 2012 Regulation of differentiation potential of human mesenchymal stem cells by intracytoplasmic delivery of coactivator-associated arginine methyltransferase 1 protein using cell-penetrating peptide. Stem Cells 30 1703-1713. (doi:10.1002/stem.1146)

Joyce JG, Tung JS, Przysiecki CT, Cook JC, Lehman ED, Sands JA, Jansen KU \& Keller PM 1999 The L1 major capsid protein of human papillomavirus type 11 recombinant virus-like particles interacts with heparin and cellsurface glycosaminoglycans on human keratinocytes. Journal of Biological Chemistry 274 5810-5822. (doi:10.1074/jbc.274.9.5810)

Kabeya $\mathrm{Y}$, Mizushima N, Ueno T, Yamamoto A, Kirisako T, Noda T, Kominami E, Ohsumi Y \& Yoshimori T 2000 LC3, a mammalian homologue of yeast Apg8p, is localized in autophagosome membranes after processing. EMBO Journal 19 5720-5728. (doi:10.1093/emboj/19.21.5720)

Kameyama S, Horie M, Kikuchi T, Omura T, Tadokoro A, Takeuchi T, Nakase I, Sugiura Y \& Futaki S 2007 Acid wash in determining cellular uptake of Fab/cell-permeating peptide conjugates. Biopolymers $\mathbf{8 8}$ 98-107. (doi:10.1002/bip.20689)

Kim K, Kim H \& Lee D 2009 Site-specific modification of genome with cellpermeable Cre fusion protein in preimplantation mouse embryo. Biochemical and Biophysical Research Communications 388 122-126. (doi:10.1016/j.bbrc.2009.07.132)
Kimura S, Noda T \& Yoshimori T 2007 Dissection of the autophagosome maturation process by a novel reporter protein, tandem fluorescenttagged LC3. Autophagy 3 452-460.

Klionsky DJ, Cuervo AM \& Seglen PO 2007 Methods for monitoring autophagy from yeast to human. Autophagy 3 181-206.

Kwon SJ, Han K, Jung S, Lee JE, Park S, Cheon YP \& Lim HJ 2009 Transduction of the MPG-tagged fusion protein into mammalian cells and oocytes depends on amiloride-sensitive endocytic pathway. $B M C$ Biotechnology 9 73. (doi:10.1186/1472-6750-9-73)

Lee JE \& Lim HJ 2012 LDP12, a novel cell-permeable peptide derived from L1 capsid protein of the human papillomavirus. Molecular Biology Reports 39 1079-1086. (doi:10.1007/s11033-011-0834-y)

Lee JE, Oh HA, Song H, Jun JH, Roh CR, Xie H, Dey SK \& Lim HJ 2011 Autophagy regulates embryonic survival during delayed implantation. Endocrinology 152 2067-2075. (doi:10.1210/en.2010-1456)

Mizushima N, Yamamoto A, Matsui M, Yoshimori T \& Ohsumi Y 2004 In vivo analysis of autophagy in response to nutrient starvation using transgenic mice expressing a fluorescent autophagosome marker. Molecular Biology of the Cell 15 1101-1111. (doi:10.1091/mbc.E03-09-0704)

Mizushima N, Yoshimori T \& Levine B 2010 Methods in mammalian autophagy research. Cell 140 313-326. (doi:10.1016/j.cell.2010.01.028)

Morris MC, Vidal P, Chaloin L, Heitz F \& Divita G 1997 A new peptide vector for efficient delivery of oligonucleotides into mammalian cells. Nucleic Acids Research 25 2730-2736. (doi:10.1093/nar/25.14.2730)

Nakase I, Niwa M, Takeuchi T, Sonomura K, Kawabata N, Koike Y, Takehashi M, Tanaka S, Ueda K, Simpson JC et al. 2004 Cellular uptake of arginine-rich peptides: roles for macropinocytosis and actin rearrangement. Molecular Therapy 10 1011-1022. (doi:10.1016/j.ymthe.2004.08.010)

Oh HA, Kwon S, Choi S, Shin H, Yoon KH, Kim WJ \& Lim HJ 2013 Uncovering a role for endocannabinoid signaling in autophagy in preimplantation mouse embryos. Molecular Human Reproduction 19 93-101. (doi:10.1093/molehr/gas049)

Peitz M, Jager R, Patsch C, Jager A, Egert A, Schorle H \& Edenhofer F 2007 Enhanced purification of cell-permeant Cre and germline transmission after transduction into mouse embryonic stem cells. Genesis 45 508-517. (doi:10.1002/dvg.20321)

Sasagawa T, Pushko P, Steers G, Gschmeissner SE, Hajibagheri MA, Finch J, Crawford L \& Tommasino M 1995 Synthesis and assembly of virus-like particles of human papillomaviruses type 6 and type 16 in fission yeast Schizosaccharomyces pombe. Virology 206 126-135. (doi:10.1016/ S0042-6822(95)80027-1)

Schwarze SR, Ho A, Vocero-Akbani A \& Dowdy SF 1999 In vivo protein transduction: delivery of a biologically active protein into the mouse. Science 285 1569-1572. (doi:10.1126/science.285.5433.1569)

Tang Y, Lin CJ \& Tian XC 2011 Functionality and transduction condition evaluation of recombinant KIf4 for improved reprogramming of iPS cells. Cellular Reprogramming 13 99-112. (doi:10.1089/cell.2010.0072)

Wadia JS, Stan RV \& Dowdy SF 2004 Transducible TAT-HA fusogenic peptide enhances escape of TAT-fusion proteins after lipid raft macropinocytosis. Nature Medicine 10 310-315. (doi:10.1038/nm996)

Wang H \& Dey SK 2006 Roadmap to embryo implantation: clues from mouse models. Nature Reviews. Genetics 7 185-199. (doi:10.1038/nrg1808)

West MA, Bretscher MS \& Watts C 1989 Distinct endocytotic pathways in epidermal growth factor-stimulated human carcinoma A431 cells. Journal of Cell Biology 109 2731-2739. (doi:10.1083/jcb.109.6.2731)

Yang Z \& Klionsky DJ 2010 Mammalian autophagy: core molecular machinery and signaling regulation. Current Opinion in Cell Biology 22 124-131. (doi:10.1016/j.ceb.2009.11.014)

Zhang H, Ma Y, Gu J, Liao B, Li J, Wong J \& Jin Y 2012 Reprogramming of somatic cells via TAT-mediated protein transduction of recombinant factors. Biomaterials 33 5047-5055. (doi:10.1016/j.biomaterials.2012. 03.061)

Received 13 May 2013

First decision 28 May 2013

Revised manuscript received 2 June 2013

Accepted 6 June 2013 